\title{
Understanding the Role of Technology to Support Breastfeeding
}

\author{
Kymeng Tang \\ kymeng.tang@kuleuven.be \\ KU Leuven, Department of Computer Science \\ Leuven, Belgium \\ Luc Geurts \\ luc.geurts@kuleuven.be \\ KU Leuven, Department of Computer Science \\ Leuven, Belgium
}

\author{
Kathrin Gerling \\ kathrin.gerling@kuleuven.be \\ KU Leuven, Department of Computer Science \\ Leuven, Belgium \\ Katta Spiel \\ katta.spiel@tuwien.ac.at \\ Human-Computer Interaction Group, TU Wien \\ Vienna, Austria
}

\begin{abstract}
Breastfeeding brings benefits for newborns and parents, but can be a challenging process. In this paper, we leverage a mixed-methods approach that builds on the Integrated Behavioural Model (IBM) to explore parents' perspectives toward breastfeeding along with their lived experiences, and examine the role of technology in this setting. Results of twelve semi-structured interviews and 175 online survey responses suggest generally positive attitudes toward breastfeeding and good theoretical knowledge. This is combined with a complex lived experience of breastfeeding where main challenges are situated in practical, emotional, and environmental/societal aspects, which are currently not sufficiently recognised by technology that seeks to support breastfeeding. Building upon our findings, we present points for reflection for the design of technology to support breastfeeding, focusing on the importance of drawing from the lived experience of parents, and ensuring that technology not only casts breastfeeding as an individual but also as a collective effort.
\end{abstract}

\section{CCS CONCEPTS}

- Human-centered computing $\rightarrow$ User models.

\section{KEYWORDS}

Breastfeeding; Integrated Behavioral Model

\section{ACM Reference Format:}

Kymeng Tang, Kathrin Gerling, Luc Geurts, and Katta Spiel. 2021. Understanding the Role of Technology to Support Breastfeeding. In CHI Conference on Human Factors in Computing Systems (CHI '21), May 8-13, 2021, Yokohama, Japan. ACM, New York, NY, USA, 13 pages. https://doi.org/10.1145/ 3411764.3445247

\section{INTRODUCTION}

Breastfeeding brings both long- and short-term benefits for newborns and mothers, but it is often reported as a challenging process [69]. The global exclusive breastfeeding rate for children aged

Permission to make digital or hard copies of all or part of this work for personal or classroom use is granted without fee provided that copies are not made or distributed for profit or commercial advantage and that copies bear this notice and the full citation on the first page. Copyrights for components of this work owned by others than ACM must be honored. Abstracting with credit is permitted. To copy otherwise, or republish, to post on servers or to redistribute to lists, requires prior specific permission and/or a fee. Request permissions from permissions@acm.org.

CHI '21, May 8-13, 2021, Yokohama, Japan

(c) 2021 Association for Computing Machinery.

ACM ISBN 978-1-4503-8096-6/21/05 . .\$15.00

https://doi.org/10.1145/3411764.3445247 less than six months is only $40 \%$, and only 23 countries in the world have a breastfeeding rate above $60 \%$ until children reach the age of six months [53], falling behind the World Health Organisation directives [54]. Various attempts to tackle barriers to breastfeeding through technology have been made, for example, through information systems that provide breastfeeding education $[1,25,31,34,36,42]$, communication systems to deliver ad-hoc breastfeeding support $[20,21,29,59,71]$, and social networks to facilitate practical and peer support [6, 16, 19, 66-68].

However, a recent review [65] of existing information and communication technology (ICT) systems that support breastfeeding revealed limited effectiveness in terms of improvement of breastfeeding rates and/or duration, and also highlights a lack of involvement of parents in the design and evaluation of such systems. This suggests a limited understanding of how ICT-based systems to support breastfeeding aligns with parents' needs and values. We set to address this gap through an enquiry into parents' lived experience of infant feeding and the role of digital technology in this space. Our work is inspired by [32] who investigated the potential of games and play to encourage breastfeeding through an exploratory study. Here, we aim to answer the following research questions (RQs):

RQ1: What is parents' anticipated or lived experience of infant feeding, and what are implications for the role of technology to support breastfeeding?

RQ2: What are parents' general perspectives on technology to support breastfeeding, how do they align with their preferences and needs across the feeding journey?

We leverage the Integrated Behavioural Model (IBM) [49] to guide our two-step research process, in-depth exploratory interviews, and an online survey building on interview findings. The IBM outlines predictors of health behaviour with a strong emphasis on intention and its relationship with the behaviour. We use this framework to elicit parents' responses toward breastfeeding. We extend the IBM with questions to explore parents' lived experience of infant feeding, and their perspectives toward technology. Results of interviews with eight parents, two parents-to-be, and two health care professionals suggest that participants have a good theoretical understanding of breastfeeding but still encounter unexpected challenges in early postnatal stages, that technology finds its way to facilitate practicalities, but concerns were raised about privacy and credibility. Survey responses from 175 participants support the notion that parents understand breastfeeding theoretically, but in 
some instances struggle with the complexity of the lived experience, highlighting a need for more supportive environmental and societal structures. Results also suggest that digital technology integration in this space is widely accepted, but that concerns about the credibility and utility of technology remain. Based on these findings, we reflect on design opportunities for technology to support breastfeeding that extend beyond the current focus on mothers as individual stakeholders, and that also take into account the lived experience of breastfeeding as reported by parents.

Our work makes the following contributions: (1) we provide a structured enquiry into breastfeeding and the role of technology, and we show how the IBM can be leveraged in this setting, (2) we show that the lived experience of breastfeeding is messy, and that technology needs to support this notion, (3) we highlight future opportunities for HCI research that aligns with parents' needs, taking into account personal, environmental and societal factors.

\section{BACKGROUND}

Here, we give an overview of barriers and facilitators to breastfeeding and existing work that provides technology to address these issues. We also summarise the Integrated Behaviour Model, the theoretical foundation for our work, and we situate it within the space of models for health human behaviour.

\subsection{Breastfeeding Barriers and Facilitators}

Many factors contribute to low breastfeeding rates; not all of them within the control of parents or healthcare providers: socioeconomic backgrounds of parents [2, 47, 52, 63], complications during delivery of the baby [43], and physical un-availability of parents or child due to medical conditions [63] are all negative predictors of breastfeeding. Beyond these issues, the success of breastfeeding is determined by one's intention to initiate the process $[23,48,58]$ and the ability to maintain breastfeeding over time [5, 8, 9, 30, 46, 47, 55]. Here, aspects such as attitudes toward breastfeeding, acknowledgement of the benefits of human milk over formula, norms, and perceived support direct the intention to breastfeed [47, 60]. Likewise, breastfeeding knowledge and skill can have a lasting impact on parents' decisions to continue to breastfeed, for example, impacting perceived self-efficacy and the ability to deal with practical issues [8]. Here, self-efficacy is related to breastfeeding experience right after the delivery, but also past experience and exposure to breastfeeding $[47,60]$. Other stakeholders also play a role as parents' perception of being supported improves both self-efficacy and intention to breastfeed [47, 64]. Beyond healthcare providers who can offer professional support, the involvement and support of partners heavily influence the decision to breastfeed and maintaining the feeding practice [64]. Insight into these barriers and facilitators helps direct the development of inquires to probe the lived experience of infant feeding.

\subsection{Technology to Support Breastfeeding}

There is a wealth of systems providing breastfeeding support. A recent review [65] categorised these systems into four categories: 1) tech-mediated breastfeeding education, 2) systems that encourage breastfeeding, 3) communication technology (e.g., video call), and 4) systems that provide practical support. Researchers in the HCI community have examined the potential of technology to address challenges for breastfeeding parents at a pragmatic level and to improve the understanding of the feeding process. Regarding pragmatic support, prior work investigated the design implications for technology to tackle logistic and environmental barriers to breastfeeding. For example, through the Feed Finder app that allows breastfeeding parents to find and review public breastfeeding places [6], the Milk Matters app that facilitates the logistics of breast milk donation [67], the Feedpal chatbot that provides answers to concerns about the feeding process [70], and through participatory design that explores desirable breast pump designs [22]. Prior work also looked into the opportunity to leverage playful technology to improve the understanding of breastfeeding among relevant stakeholders [32]. Beyond breastfeeding, the HCI research community has also investigated technology design for parenthood more broadly. For example, existing work provides an investigation into peer support seeking among pregnant women [35], the role of ICT in supporting new mothers [33] and the disclosure of pregnancy loss on social media [4]. Here, prior work reveals an extensive use of technology among parents in exploring their (new) identity, reassurance, and emotional support from peers, hence, suggesting future research should focus on community efforts to support parenthood. While these studies illustrate the opportunity for technology design to support parenthood and breastfeeding, no structured analysis of its potential in supporting breastfeeding has been carried out to date. Our work addresses this gap by investigating the role of technology to support breastfeeding more broadly through an inquiry into parents' lived experiences of breastfeeding across the feeding journey from antenatal to postnatal stages.

\subsection{Integrated Behavioural Model}

The integrated behavioural model (IBM) [49] extends the theory of reasoned action (TRA) and the theory of planned behaviour (TPB), describing five main predictors of a health-related behaviour (see Figure 1). In the TRA, Fishbein [27] described that attitudes toward a behaviour and subjective norms direct the intention which is an important predictor of the behaviour performance. Ajzen [3] extended the TRA by including perceived control over a behaviour as another direct determinant of behavioural intention, hence, creating the TPB. Building upon the TRA and TPB, the IBM emphasises on the behavioural intention as the key predictor of a behaviour and also integrates other direct behaviour enablers: knowledge, environmental constraints, salience, and habit.

In the context of breastfeeding, the IBM lines up well with literature concerning breastfeeding determinants. For example, parents' positive breastfeeding attitude is associated with the act of nurturing their children [40] (experiential attitude) and the belief that breastfeeding is beneficial for health [60] (instrumental attitude). Breastfeeding support from partners, family members and friends, and health care professionals $[47,60]$, is a part of a descriptive norm while an injunctive norm is associated with societal norms about breastfeeding [60]. Descriptive and injunctive norms together form a perceived norm construct, which reflects the pressure to breastfeed or to bottle feed. The personal agency construct describes parents' perceived ability to breastfeed, includes confidence in their 


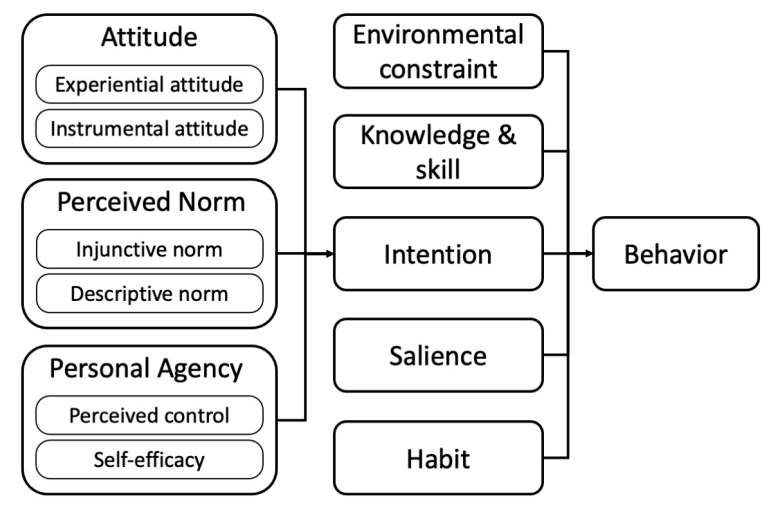

Figure 1: The integrated behavioural model

ability [8] (self-efficacy) and their perceived control over the environmental factors that allow them to breastfeed [44]. Parents may breastfeed if they were equipped with breastfeeding knowledge and skill $[8,39]$, not having environmental constraints that prevent them from breastfeeding (e.g., workplace policy [52]), and know the salience of breastfeeding [60].

The IBM has an edge over other health behaviour theoretical frameworks when used as a requirements elicitation tool. Compared to relevant models such as the Information-Motivation Behavioural skills model [28], the Self-Determination Theory [62], and the Social-Cognitive Theory [7], the IBM is more complete and rigorous as it focuses not only on factors that influence the intention to breastfeed but also the factors that enable parents to proceed. Although models like the Health Belief Model [61] and the Transtheoretical Model [57] also describe the driving forces behind certain health behaviours, they are less suitable here as they tend to characterise feeding options other than breastfeeding as an unhealthy behaviour that should be altered. The relevance of the IBM allows us to fully capture motives to breastfeed (or bottle feed) among parents and parents-to-be, which will, in turn, indicate design implications for breastfeeding technology. The model has been extensively used to understand the behaviour of HIV/AID preventions [37], to predict high-risk drinking behaviours [13], and to identify the roles and design goals of technologies for wheelchair users [45].

\section{PHASE 1: INTERVIEW STUDY}

In the first phase of our work, we carried out semi-structured interviews with parents (8), parents-to-be (2), and health care professionals working with parents (2) to better understand parents' lived and anticipated experiences of breastfeeding, and their perspective on technology in this space.

\subsection{Method}

We invited participants to take part in semi-structured interviews about their motives to breastfeed or bottle feed (with formula) with questions structured around the IBM constructs, for example, "How do you see breastfeeding in general?" (attitude), "Do you feel the pressure to breastfeed to be perceived as a good parent?" (perceived norm), and "What are the most significant benefits and drawbacks of breastfeeding for you and your children?" (personal agency and salience). We further probed their lived infant feeding experience through questions like "Can you briefly describe your infant feeding in a timely manner?". Here, we invited parents who predominantly bottle feed their child to clarify whether they bottle fed with formula or breastmilk. We use the term bottle feeding to describe bottle feeding with formula throughout this paper, as all participants reported bottle feeding as the use of formula. We also elicited their perception and use of technology in infant feeding context with questions such as, "Do you use or plan to use any technology (e.g., apps \& websites) to support your infant feeding practice? Can you elaborate more about your reasoning?" and "What do you think about the use of technology to support infant feeding?".

\subsection{Procedure}

Participants were recruited through a snowball sampling method with advertisements posted on social media, and through wordof-mouth between June 2019 and October 2019. Each participant took part in a semi-structured interview of about one hour at their preferred venue. The interview covered two main topics: infant feeding practices, and the use of technology in that space. The questions were about their perspectives on both breastfeeding and bottle feeding, their practice and associated challenges of the two modes of feeding, their coping strategies, the use of technology in infant feeding and general context, and their perceptions thereof. The interviews were conducted in English, Dutch, and Khmer. NonEnglish interview transcripts were translated to English by fluent speakers. Participants were compensated with a cash payment of 20 euros or a gift card for a shop of their choice of the same value.

\subsection{Participants}

Twelve participants (nine women and three men; eleven in the 26-35 age group and one in the 18-25 age group) took part in the interviews individually. Among them, two were health care professionals (one paediatrician and one midwife), two expecting mothers, one expecting father, two parents who predominately breastfed their children, two parents who predominately bottle feed, one bottle feeding parent, and two partners of a breastfeeding parent. Across the parents, their children were on average 10.49 months old ( $\mathrm{SD}=6.536)$. Twelve participants are based in western Europe (eleven in Belgium, one in France) but grew up in different countries (nine from Belgium, one from Cambodia, one from Greece, and one from Russia). Participants use a broad range of technology and social media channels. Participants hold a Master's degree or higher (10) and a high-school diploma (1).

\subsection{Data Analysis}

We combine an exploratory, inductive approach with a deductive approach: using the deductive approach, a share of the data was coded independently by two members of the research team, and initial codes were discussed to determine agreement/disagreement [11] and resolve possible issues in the interpretation of the IBM constructs. The remaining data were then coded by the main researcher. The inductive analysis follows Braun and Clarke's reflexive thematic analysis [15]. Here, data were read and re-read by the main 
researcher, then coded, and aggregated into initial themes around parents' lived experiences of infant feeding and their perspectives on technology. The themes were then reviewed within the research team. In total, 53 codes, which were refined throughout the coding process, were assigned to 1505 data points. From these codes, three main themes were crafted.

\subsection{Results}

Here, we summarise our findings, coded in accordance with the IBM's constructs (attitudes, perceived norm, personal agency, environment, knowledge, salience and habit), and three themes crafted during the inductive analysis of our data: becoming a resilient infant feeder, the emotional toll of breastfeeding, technology deployment in a sensitive setting.

3.5.1 Attitude. Attitude towards breastfeeding as expressed by the breastfeeding parents were predominantly positive, and mainly relate to general health benefits and an intimate parent-child bond, e.g., "it's also something is really linked to a very healthy bonding with the child as well, a very intimate experience [...] health benefits for the child but also for the mom" (P6, predominately bottle feeding parent). Negative perspectives were related to the image of breastfeeding, e.g., "I still have the image of the breastfeeding mom are more like the hippy style. [...] I couldn't imagine myself doing it; [...] I was not considered doing the pumping; that's too cow like" (P8, bottle feeding parent) and concerns around pain and difficulty, e.g., "I'll not go through hell to give the breastfeeding" (P12, expecting parent). Interestingly, participants regarded bottle feeding as a luxury option, but a better way to share parental responsibility. For many, it was a fallback option if breastfeeding failed. Attitudes of partners of breastfeeding parents were more neutral. While interviews revealed that partners sought to be supportive (e.g., providing encouragement or taking over chores to relieve the breastfeeding parent), we observed one instance where the partner was in favour of bottle feeding, with the breastfeeding partner stating "To him, the only way to fix [problems with breastfeeding] is to bottle feed" (P2).

3.5.2 Perceived Norm. Perceived norm was pervasive throughout all interviews, with patterns revolving around social pressure with respect to the mode of feeding. Parents expressed concern over the pressure to breastfeed among family and friends, e.g., "[...] you must be breastfeeding because everybody breastfeeds and breastfeeding is what you should do" (P8), and "They might be judgemental but not in a very vocal way" ( $\mathrm{P} 9$, expecting parent). Likewise, healthcare professionals and hospital environments were seen as a source of pressure, and participants reported negative experiences on social media, referring to these stakeholders as the "breastfeeding mafia". Here, social media appeared to increase pressure rather than alleviate it, e.g., "[...] a few young mothers that I follow on Instagram or on youtube [...] switched to bottle, the comments are so cruel and mean that you really feel that hmm, wow it's not ok to give a bottle" (P8). This notion was supported by healthcare professionals, who suggested that parents associate guilt with bottle feeding even when there was a medical indication for switching from breastfeeding: "But if I say like ok, bottle feeding, they feel directly guilty." (P7, midwife). Perceived norms also extend beyond the choice of whether to breastfeed to societal norms on how to breastfeed. Notably, one mother commented that breastfeeding beyond the baby days was challenging norms, stating that "some moms, who want to breastfeed longer than 4 to 6 months, feel almost weird, ... because people might give them comments, like why do you still breastfeed your child after 6 months" (P11, expecting parent). Likewise, breastfeeding in public provoked conflicting responses, with most participants agreeing that parents can breastfeed anywhere, but should do it discreetly. For example, one participant pointed out that "let's say in a metro, and I'm sitting next to you. Hmm, this has happened to me, this is like come on, maybe not, maybe (breastfeeding is) something to do at home" (P9), suggesting that public breastfeeding still is associated with perspectives that directly contradict guidelines for breastfeeding on demand [12,50].

3.5.3 Personal Agency. Personal agency - consisting of perceived control and self-efficacy construct - has a strong presence in our data, with instances predominantly addressing the relationship between infant feeding, agency, and implications of the lack thereof. Positive instances of perceived control were mainly reflected in the convenience associated with each of the feeding modes: most participants found breastfeeding a convenient option, e.g., "you don't have to carry a lot of stuff with you" (P1) and "you always have it with you, and it is always at the right temperature" (P3). Bottle feeding parents highlighted how sharing parental responsibility contributed to perceived control, suggesting that it meant they would not have to "lock myself in the house to not coming out anymore" (P12). This was also reflected in a perceived lack of agency expressed by breastfeeding parents, e.g., "[the baby] is kind of tied up to me, daddy cannot take care of him" (P1). Interestingly, one partner expressed jealousy over their inability to breastfeed their child, suggesting that they felt "jealous that I will never be able to experience towards my children what my wife experiences" (P5). Feeding modes also had an impact on self-efficacy. Here, bottle feeding parents felt more certain of their routines, with one participant suggesting that "the baby comes with instructions" (P8), whereas milk supply management affected the perceived self-efficacy of breastfeeding parents and caused parents to stop breastfeeding (P2). Generally, this theme suggests that continuously responding to the needs of the baby reduced parents' personal agency, and that breastfeeding had more challenging implications in terms of parental lifestyle and mothers' perceived control in particular.

3.5.4 Environment. Environmental constraints for breastfeeding are strongly related to the professional careers of parents. Many believe that their breastfeeding practice is determined by the length of their parental leave, hence, perceiving returning-to-work as a major barrier for breastfeeding. Here, a healthcare professional echoed that parents "feel bad about having to ask for [breastfeeding] breaks, and have the impression that they have to stop because they start working again" (P11). Participants further commented that work environments play an important role in supporting or discouraging breastfeeding, e.g., "a good thing with my job, I can manage my own time, definitely try to hmm, do it for as long as I can" (P11) and "I'm constantly working in lab or meeting, so to plan it, for me it's a disaster" (P12). In relation to that, a lack of breastfeeding facilities at work poses another barrier for parents to continue breastfeeding. There are a few instances that indicate the 
entwined relation between environmental constraints and other arms of IBM: personal agency and perceived norm. Instances here show that the environmental constraints of breastfeeding are rather the perception of control and self-efficacy; a few participants believe "that's what you see is possible.[...] Because everybody is allowed to pump, it's regulated by law" (P7). Likewise, our findings suggest that culture and social norms can contribute to the perceived barriers. For instance, a Cambodian participant stated that "We can see [breastfeeding mothers] everywhere because we don't discriminate or judge them. But in Europe, I think they are very shy. [...] They need to find a quiet place, very discreet and don't want other people to know what they do" (P10).

3.5.5 Knowledge. Most participants expressed that they have enough theoretical understanding of breastfeeding with respect to the technicalities (e.g., feeding cues, latching, on demand feeding), obtained through various channels (e.g., antenatal classes, websites of healthcare providers). However, participants also expressed various insecurities about their feeding practice (e.g., whether their child received enough milk), suggesting a difference between theoretical knowledge and practical proficiency and confidence in the feeding method. With respect to breastfeeding information, some participants commented that materials idealised the image of breastfeeding while insufficiently addressing potential challenges and coping strategies (P2, P6). Participants also commented that maternal education dismissed bottle feeding (P5, P6). While healthcare professionals argued that they are in the position to inform parents about the process without pressuring parents to breastfeed (P7, P10), these findings suggest that broadening parents' knowledge of breastfeeding with respect to challenges, and adequately addressing bottle feeding would prepare them more adequately for infant feeding.

3.5.6 Salience. Participants generally acknowledge the importance of breastfeeding. Participants are concerned about the health benefits for breastfeeding parents and their children, and the parentchild bond established through the process of feeding. Quotes from parents include "the interaction of giving bottle doesn't have skin to skin contact, and intimacy" (P10), "breastfeeding is healthy" (P1), "the bonding part with the baby" (P6), and "good for [my child's] health" (P5). Partners were also aware of these benefits, with one father expressing that "you get some sort of resistance from the mother passed on to the child" (P4) and "it's a bonding moment" (P9). Interestingly, all parents used the language of healthcare providers and information materials about breastfeeding; here, we can conclude that participants were well-aware of the benefits of breastfeeding.

3.5.7 Habit. Our data suggests that habit is a difficult construct in the context of breastfeeding: especially parents with newborns may struggle to establish routines and are in fact encouraged by healthcare providers to dynamically respond to their children's needs. Regardless, participants often conceptualised the idea of habit as establishing a schedule, and then had to concede that they find their children's demand unpredictable (P1, P8). In terms of adopting routines, there were a few instances where parents developed a pumping schedule to adapt their milk supply (P3, P4, P5, P6) and to be able to bottle feed (using breast milk) in public places (P3, P5, P12). In terms of general breastfeeding habits, parents further elaborated that they typically breastfeed in private spaces $(\mathrm{P} 1, \mathrm{P} 2, \mathrm{P} 3, \mathrm{P} 4, \mathrm{P} 5, \mathrm{P} 6)$ or discreetly in public if they have to (P3, P5), e.g., "I'm not going to sit in the middle of a restaurant [...] I always tried to do it discretely" (P3). Our data contained no instances of parents commenting on the general impact of habit, e.g., having breastfed the first child and simply falling into the same pattern with the second child.

3.5.8 Parent's Lived Experiences and Views on Technology. We applied inductive Thematic Analysis [14] and crafted three themes from our data, spanning the need to (1) become a resilient infant feeder, (2) the emotional toll of breastfeeding, and (3) technology deployment in a sensitive setting.

Theme 1: Becoming a Resilient Infant Feeder. Our data reveals the importance of resilience in the context of infant feeding in general, and breastfeeding in particular.

Managing the Feeding Process. Parents generally described their infant feeding journey as a mission to satisfy their child's needs while seeking to maintain their own health and comfort. This includes the ad-hoc development of coping strategies when feeding becomes challenging. Participants noted that they had to embrace the fact that "every baby is different" (P5) and that you "can't be for $100 \%$ prepared" (P12). To adapt to the unpredictability of breastfeeding, most participants combined preemptive and responsive strategies. Preemptively, parents reported trying to "be as realistic as possible not to be disappointed" (P12) and avoid having "a fixed idea in your head about I want to do this for this long or I want it like this". Parents' responses to unforeseen challenges are strongly linked with technology use. Some parents turned to online communities to relate to others' stories of breastfeeding experiences and to see how other people cope with challenges (P4), or follow up on other people's choice of feeding practice after encountering similar challenges (P2). Web searches regarding specific issues (rather than general breastfeeding information) also played an important role. Participants reported using a range of technology platforms to log their feeding routines in the early days, e.g., "Google home [...] configured that so it put on an excel sheet" (P1) and mobile apps (P1, P3, P4, P6, P7, P8), helping them to understand their feeding habits, to establish new routines, and also offered reassurance that their child was well-fed.

Managing other stakeholders. Our findings suggest that there are many instances in which parents have to cope with other stakeholders. Many breastfeeding participants suggested that their partners remained silent on the topic of breastfeeding, or did not expect them to breastfeed, but in consequence also were uninvolved in decision-making. Here, feeding parents felt burdened by having to decide on the feeding mode on their own. In contrast, extended family members were vocal; participants shared instances where parents-(in law) of breastfeeding parents actively encouraged bottle feeding, e.g., suggesting that "you would not be the only one, and your baby would be healthy" (P2), or when practices within extended family led to contradictory expectations: one participant explained that "In my family [...] there was some expectation to breastfeed" whereas the parents-in-law "have lived in the time where breastfeeding wasn't that common." (P4). When asked to reflect on their 
general strategies for managing the expectations of others, a number of parents suggested that they eventually gave in and adjusted feeding strategies, while others followed their path.

Theme 2: The Emotional Toll of Breastfeeding. This theme reflects the emotional challenges that parents experienced throughout the feeding journey that often oscillates between moments of intimacy and wonder, and exhaustion and frustration. Breastfeeding participants reported instances of intimacy, and described feeling "amazed" (P3, P5) and "special" (P7, P6). Partners sometimes offered a more nuanced perspective, with one father suggesting that "[his partner breastfeeding his child] is one of the most beautiful processes that I witnessed in person, but it is also rough and painful" (P5). Practically, negative emotions were often related to physical discomfort. For example, breastfeeding was described as "difficult", "not enjoyable" and "frustrating" due to unexpected challenges (P6, P2). Likewise, breastfeeding parents expressed conflicting feelings about the feeding relationship with their child, often linked to their own exhaustion, e.g., "[...] at night, it would be easier if she would find her comfort in something else" (P3) and "but yeah, 3 in the morning, it's a chore, [smiled]" (P8). We also observed instances where bottle feeding parents expressed jealousy at parents who did succeed in breastfeeding, and partners who, as a result of breastfeeding felt less involved and experienced negative emotions: "[...] I do feel in a way jealous that I will never be able to experience towards my children what my wife experiences [...]" (P5) and "I think that as a father it's, you're sort of at the sideline." (P4). Some participants reported feeling emotionally burdened by these experiences (P3, P2) and ultimately stopped breastfeeding because of them (P2, P6). However, the transition to bottle feeding also created challenges. For instance, one participant noted that it was "so emotional, it was a really hard decision to make, to stop, in my time, it was a good decision, but it was really hard to make that decision" (P2) and "I felt guilty, although I know I shouldn't feel that way, but I did." (P6). The end of the breastfeeding journey also took an unexpected emotional toll on some breastfeeding parents, feeling emotional about releasing the bond they had built with their child.

Theme 3: Technology Deployment in a Sensitive Setting. Generally, attitudes toward technology to support infant feeding were positive, and parents used a variety of systems that were often not specifically designed to support breastfeeding (e.g., use of home assistants to track feeds). However, there also were instances where participants raised concerns about technology, which we discuss in more detail here.

Many participants were concerned about the credibility and their interpretation of information provided through technology. On the surface, participants mentioned the challenges of evaluating the credibility of online information, and trying to limit themselves to reputable health organisations. Some participants mentioned that trying to access information in English posed another barrier, for example, they worried about translation errors (P10). Particularly regarding interactive systems to provide information, participants were curious about simulations and training systems to allow them to better prepare themselves for infant feeding. However, participants also worried that such systems might not provide a realistic image of breastfeeding and dismiss potential challenges, echoing general concerns about breastfeeding education and information. Likewise, one participant was negative about playful technology, suggesting that it would not be adequate in this setting: "If I would have to decide baby based on let say a Tamagochi, taking care of the animal, in that case a baby, the answer would for sure, would be no" (P12).

A number of participants expressed worry about privacy, and suggested they felt vulnerable particularly if technology involved their newborn baby. Participants reported that they were afraid of "being tracked" (P8) and criminal activities (P12). However, other participants dismissed these concerns, e.g., "Google is also very invasive of my privacy but that has a benefit: the services they offer [...] if it is just collecting my data to sell to someone else, then I wouldn't use the technology." (P4). Privacy concerns also influenced participants' choice of technology, for example, when purchasing a baby monitor, one participant reported "choose a basic one than the one with the camera and everything, because you see with criminal mind thing, stalker, steal baby [laugh] [...] I don't want them to put any like, dozen of pictures of the baby on the social media" (P12). Here, it is interesting to note that the perceived threat was very high; generally, we observed heightened awareness of security and privacy concerns that may otherwise be dismissed by the general population [17].

\subsection{Summary of Findings}

The results of the interview study suggest that participants generally had positive attitudes toward breastfeeding, were aware of its importance, and had good theoretical knowledge about the technicalities of the feeding process. However, some participants felt that practical issues (e.g., establishing habits, addressing unexpected challenges, and navigating environmental challenges) were insufficiently addressed by breastfeeding education, and resulted in a mismatch between their expectations and the lived experience of breastfeeding. Here, parents highlighted the importance of becoming a resilient infant feeder who has the practical resources to dynamically tackle unexpected challenges (e.g., extensively consulting online resources to address individual feeding problems). Likewise, findings suggest that breastfeeding can be emotionally challenging due to physical demands, and that parents experience feelings of loss or guilt when breastfeeding comes to a (sometimes earlier than planned) end. Finally, attitudes toward technology and breastfeeding were positive in the context of practical support (e.g., to track feeds); however, participants expressed concerns about educational systems with respect to the quality of content regarding factual accuracy and adequate representation of challenges. Likewise, the idea of technology directly linked with children sparked privacy concerns. Generally, parents predominantly viewed technology as a tool to manage the feeding process particularly in the early stages of breastfeeding, with its importance dwindling as they grew more confident.

\section{PHASE 2: SURVEY STUDY}

In the second phase of our study, we reached out to a wider audience by deploying an online survey to follow up on the main interview findings. 


\subsection{Method}

Building on the results of the interview, we developed an online survey that consisted of 99 questions divided into four main parts: (1) Demographic information and personal infant feeding practice (age, education level, country of residence, feeding practice, and technology use). (2) Examination of the IBM constructs using 5-point Likert scales (attitude, perceived norm, personal agency, knowledge and skill, environmental constraints, salience, habit) with items such as "I don't feel very comfortable seeing people breastfeed in front of me" (Attitude), "I think I'm/my partner is skilful enough to breastfeed" (Knowledge), "I think breastfeeding is convenient" (Personal agency), and "I think breastfeeding is not as crucial as advertised" (Salience). (3) Exploration of parents' lived infant feeding experience, with questions polling for their practical and emotional experience of the feeding process, e.g., "I find it difficult to anticipate what breastfeeding would be like", and "I think breastfeeding gives an opportunity to have an intimate parent-child moment". (4) Inquiry into technology use and perspective towards technology in infant feeding space. Sample questions include: "I think technology can't convey a realistic image of breastfeeding." and "Please list the technology that you have used in the context of infant feeding". The survey included a combination of question types, with most statements to be rated on 5-points Likert scales, multiple-choice questions with an optional input field, and open questions. Depending on their role in the infant feeding process, participants received an adapted set of questions reflecting their own situation (breastfeeding parents, parents who bottle feed with formula, partners, and parents-to-be). On average, each participant would see 94 questions. Please refer to the supplementary materials for the full survey.

The research protocol was approved by the institutional ethics board. At the beginning of the survey, participants provided informed consent; the survey length was around 30 minutes depending on the amount of qualitative feedback participants provided. The survey was made available on Qualtrics from January 22, 2020 until April 1, 2020. The survey link was distributed in forms of posters, word-of-mouth through friends and colleagues, and through social media communities targeting (expecting) parents. Recruitment materials explicitly polled for the participation of non-breastfeeding parents, partners, and expecting parents.

\subsection{Data Analysis}

We received a total of 284 responses, of which 175 were identified as complete and included in our analysis. Data were analysed on Jupyter notebook using scipy.stats and scikit_posthoc Python packages. As a pre-processing step, we inverted scores for items that were phrased negatively. We provide descriptive statistics for all relevant constructs, and identify key differences between participant groups using Kruskal-Wallis [41], effect size $\left(\eta^{2}\right)$ [18] and Dunn tests [24] with Bonferroni correction. We selected these tests as a result of the limited responses from non-breastfeeding parent groups [26] (see following section for breakdown of respondents). Here, we characterise statistical significance between groups when the p-value is less than 0.05 . Qualitative data are provided to further explain quantitative findings.

\subsection{Results}

Here, we first present an overview of respondents, their feeding practice, and technology use. We then report the results for the IBM, and present outcomes of our analysis of questions about parents' lived experience of infant feeding and their perspectives towards technology in this setting.

4.3.1 Characteristics of Respondents: Feeding Routines and Technology Use. Among the 175 respondents, 153 are women, 20 men, and two non-binary. Participant age ranges from 18-25 (5), 26-36 (108), 36-45 (58) and 46-50 (4). We categorise participants into four groups: predominantly breastfeeding parents (126), parents who predominantly bottle feed with formula (bottle feeding parents) (12), partners (12 breastfeed, 5 bottle feed), and parents-to-be (20). Of the breastfeeding parents, the duration of the feeding relationship was two weeks or less (2), up to three months (4), up to six months (18), up to nine months (11), and up to one year or longer (91). Participants were located in Europe (139), North-America (29), South-East Asia (4), Oceania (1), and two participants did not disclose their country of residence.
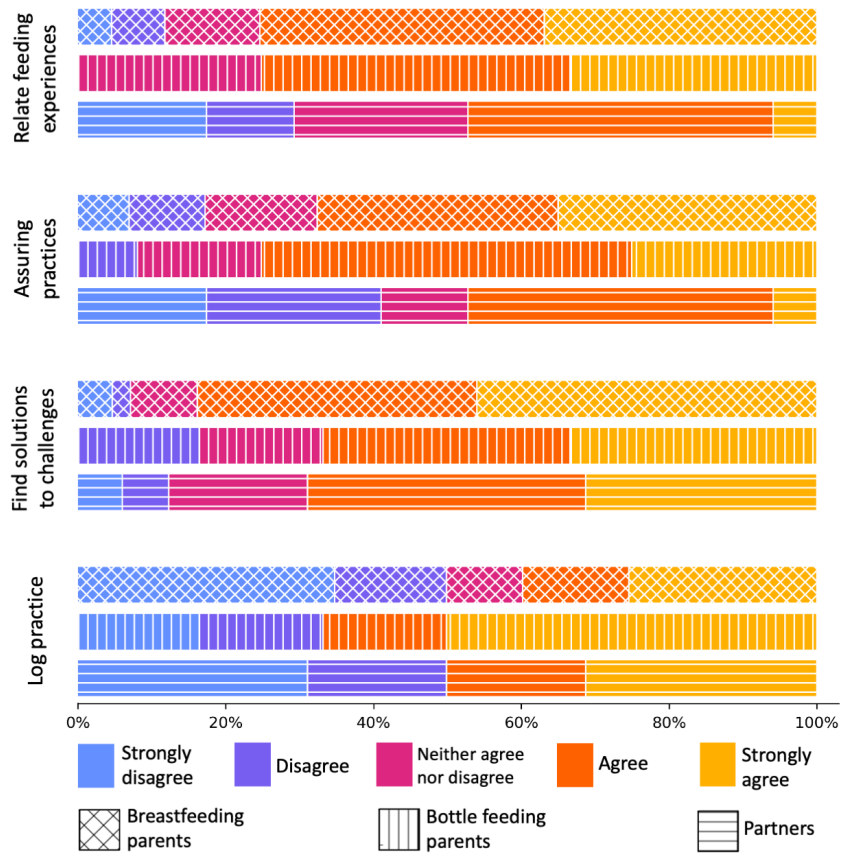

Figure 2: Distributions of the survey responses for the use of technology in infant feeding among parents rated on 5-point Likert scales ( $5=$ strongly agree)

Generally, participants reported using a wide range of technology: smartphones (172), computers (170), tablets (92), smartwatches or fitness trackers (45), gaming systems (20), mobile phones (6), and e-readers (1). Participants engage in social media and chat platforms such as Facebook (174), WhatsApp (153), LinkedIn (104), Instagram (103), Twitter (47), Snapchat (14), Discord (13), and others (12); only four participants did not use social media. Participants 
reported using a breadth of technology to support their infant feeding journey: mobile apps (BabyTracker: 6, FeedBaby: 3, Medela: 3, GrowBaby: 3, Huckleberry: 3, WhatsApp: 2, Apps to log feeding practice: 13, other: 24), social media (Facebook -groups-: 31, Reddit: 7, Youtube: 4, other: 2), breastfeeding support websites (KellyMom: 20, La Leche League: 15, Baby center: 9, Borstvoeding: 6, Kind en Gezin: 5, and other: 15), official sites for health (the NHS: 7, other: 4), other websites (9), web search (7), online forum (7), and phone call (1).

Technology was a common tool among parents to support infant feeding, with specific areas of interest varying depending on their role in the feeding process (see Figure 2). Significant differences between groups were found in the use of technology to relate infant feeding experiences $\left(\mathrm{H}_{2}=9.265, \mathrm{p}=.010\right)$ and to assure feeding practice $\left(\mathrm{H}_{2}=6.930, \mathrm{p}=.031\right)$. The use of technology to relate to the feeding experiences of others is high among breastfeeding parents (Median=4, IQR=5-4), significantly higher than of partners (Median $=3, I Q R=4-2 ; z=3.001, p=.010$ ). Likewise, breastfeeding parents use technology to assure their infant feeding practice (Median $=4$, $\mathrm{IQR}=5-3)$ significantly more than partners (Median=3, $\mathrm{IQR}=4-2$; $\mathrm{z}=2.612, \mathrm{p}=.030)$.

4.3.2 Analysis of the IBM Constructs. Here, we present the responses within each of the categories of the IBM. First, we report an overview; then we present an analysis based on groups (breastfeeding parent, bottle feeding parent, partner, expecting parent).

Attitude toward breastfeeding scores high (Median=5, IQR=5-3) across groups, with significant differences between groups $\left(\mathrm{H}_{3}=43.26\right.$, $\left.\mathrm{p}<.001, \eta^{2}=0.079\right)$. Follow-up pairwise testing reveals that breastfeeding attitude among breastfeeding parents (Median=5, IQR=5-4) is significantly higher than that of bottle feeding parents (Median=3, $\mathrm{IQR}=5-1 ; \mathrm{z}=4.481, \mathrm{p}<.001)$, partners (Median=4, $\mathrm{IQR}=5-2 ; \mathrm{z}=3.966$, $\mathrm{p}<0.001)$ and parents-to-be (Median $=4, \mathrm{IQR}=5-3 ; \mathrm{Z}=3.807, \mathrm{p}<.001$ ). No other significant differences were found.

Perceived norm, i.e., positive societal norm about breastfeeding, scores average (Median $=3, \mathrm{IQR}=4-2$ ). No significant difference between groups was found $\left(\mathrm{H}_{3}=3.9, \mathrm{p}=.272, \eta^{2}=0.001\right)$.

Personal agency with respect to breastfeeding is rated above average (Median $=4, \mathrm{IQR}=5-2$ ). However, there are significant differences between groups $\left(\mathrm{H}_{3}=77.44, \mathrm{p}<.001, \eta^{2}=0.0551\right)$. Pairwise comparisons show that personal agency is perceived as higher by breastfeeding parents (Median $=4, \mathrm{IQR}=5-2$ ) than by bottle feeding parents who score low (Median $=2, \mathrm{IQR}=4-1 ; \mathrm{z}=7.096, \mathrm{p}<0.001$ ), partners (Median=3, IQR=4-2; $\mathrm{z}=3.072, \mathrm{p}=.013$ ) and parents-to-be (Median=3, IQR=4-2; $\mathrm{z}=4.988, \mathrm{p}=.004$ ). There is also a significant difference $(\mathrm{z}=3.584, \mathrm{p}=.002)$ between partners $($ Median $=3, \mathrm{IQR}=4-2)$ and bottle feeding parents (Median $=2, \mathrm{IQR}=4-1$ ).

The impact of Environment on breastfeeding received a score of (Median=3, IQR=4-2) with no significant differences between groups $\left(\mathrm{H}_{3}=0.469, \mathrm{p}=.926, \eta^{2}=0\right)$.

Knowledge about breastfeeding scored relatively high across groups (Median $=4, \mathrm{IQR}=5-3$ ) though there are significant differences between groups $\left(\mathrm{H}_{3}=13.897, \mathrm{p}=.003, \eta^{2}=0.0113\right)$. Pairwise comparisons show that partners' scores (Median $=4, \mathrm{IQR}=5-4)$ are significantly higher $(\mathrm{z}=3.25, \mathrm{p}=.007)$ than those of parents-to-be (Median=4, IQR=4-3). No other differences were observed.
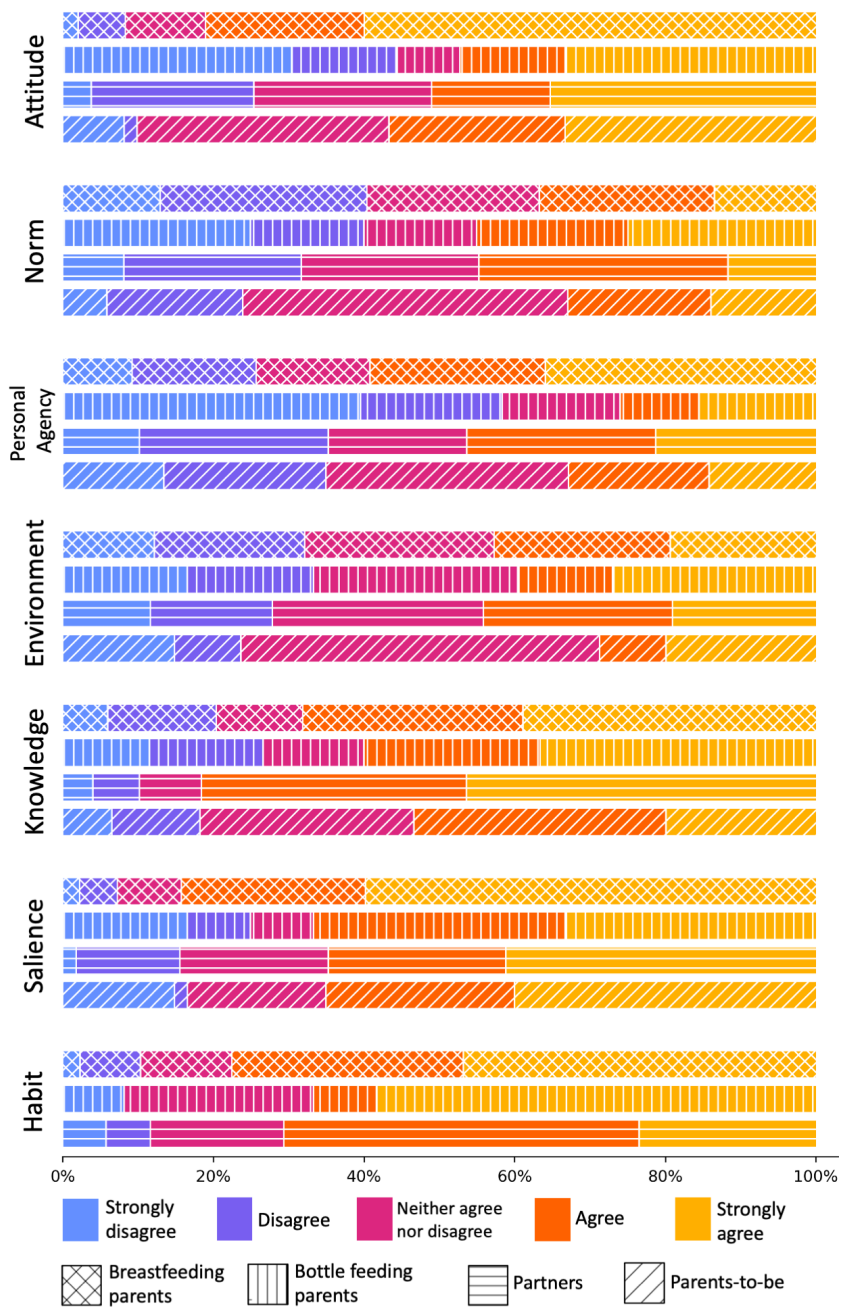

Figure 3: Distributions of the survey responses for IBM constructs with respect to perspectives on breastfeeding rated on 5-point Likert scales (5=strongly agree; Habit omitted for parents-to-be as not applicable)

Salience, i.e., awareness of the importance of breastfeeding, is rated highly (Median=5, IQR=5-4) across groups. Significant differences between groups $\left(\mathrm{H}_{3}=26.424, \mathrm{p}<.001, \eta^{2}=0.045\right)$ reveal that breastfeeding parents (Median $=5, \mathrm{IQR}=5-4$ ) are more aware of the importance of breastfeeding than bottle feeding parents (Median $=4$, $\mathrm{IQR}=5-2 ; \mathrm{z}=3.433, \mathrm{p}=.006$ ), partners (Median $=4, \mathrm{IQR}=5-3 ; \mathrm{z}=2.923$, $\mathrm{p}=.021$ ), and also parents-to-be (Median $=4, \mathrm{IQR}=5-3 ; \mathrm{z}=3.46, \mathrm{p}=.003)$. No other differences were found.

Habit also scores high across groups (Median=4, Range IQR=5-4). We did not find significant differences between groups $\left(\mathrm{H}_{2}=2.457\right.$, $\mathrm{p}=.483, \eta^{2}=0.0031$ ).

4.3.3 Parent's Lived Experience. Here, we present the responses concerning the lived experience of infant feeding.

Becoming a resilient infant feeder. Managing the feeding process: Participants appreciate that breastfeeding is a challenging task 
(Median=4, IQR=5-3) with significant differences between groups $\left(\mathrm{H}_{3}=8.816, \mathrm{p}=.032, \eta^{2}=0.0112\right)$, in particular, breastfeeding parents (Median=4, IQR=5-3) significantly perceive lower challenge than bottle feeding parents (Median $=4, \mathrm{IQR}=5-4 ; \mathrm{z}=2.96, \mathrm{p}=.019$ ). Participants also reported high levels of effort (Median=4, IQR=5-3) to get informed about infant feeding, with no significant differences between groups $\left(\mathrm{H}_{3}=5.188, \mathrm{p}=.159, \eta^{2}=0.0129\right)$. Comments reveal parents' complex responses to breastfeeding challenges, for instance, "Starting the breastfeeding proved to be more challenging than expected, as my first child had trouble latching, and [...] the milk production of my wife didn't start right away [...] So we had to make a lot of effort with additional formula feeding and intensively pumping milk in order to get everything started" (S227, partner), and frustration related to expectation issue, e.g., "it is, or was to me, such a big surprise that breastfeeding isn't just happening perfectly right from the start, but it's painful and quite difficult. I hadn't anticipated that and I think many women don't" (S37, breastfeeding parent).

Managing other stakeholders: Participants reported low levels of expectation from the non-feeding partner on their decision to breastfeed or bottle feed (Median $=2, I Q R=3-1$ ), with no significant differences between groups $\left(\mathrm{H}_{3}=2.593, \mathrm{p}=.459, \eta^{2}=0\right)$. Similarly, the perceived expectation from family members is also low (Median=2, IQR=4-1), and no between-groups differences are observed $\left(\mathrm{H}_{3}=3.908, \mathrm{p}=.272, \eta^{2}=0.0026\right)$. However, the expectation from health care professionals is reported as high $($ Median $=4, \mathrm{IQR}=5$ 3) without significant differences between groups $\left(\mathrm{H}_{3}=2.691, \mathrm{p}=.442\right.$, $\left.\eta^{2}=0\right)$. The perceived expectation from wider society is average (Median=3, IQR=4-2) with no differences between groups $\left(\mathrm{H}_{3}=7.476\right.$, $\mathrm{p}=.058, \eta^{2}=0.0263$ ). Here, comments provide examples of how this expectation manifests (and how it relates to relevant dimensions of the IBM, e.g., norm and environment), e.g., "after the first 6 months, I was more likely to get comments about still breastfeeding [...] I only nursed inside our house (mornings \& evenings) after the first year, so no need for people to feel uncomfortable there." (S76, breastfeeding parent). Generally, respondents reported satisfaction with their own feeding decisions (Median=5, IQR=5-4); no between-group differences, $\left(\mathrm{H}_{3}=2.806, \mathrm{p}=.422, \eta^{2}=0\right)$, and they report that their partner was the only other relevant stakeholder when making a feeding decision (Median=5, IQR=5-4); no between-group differences $\left(\mathrm{H}_{3}=6.74, \mathrm{p}=.081, \eta^{2}=0.0108\right)$.

Emotional toll of breastfeeding. Intimacy of breastfeeding is generally reported as high (Median $=5, I Q R=5-4)$ with significant differences between groups $\left(\mathrm{H}_{3}=13.169, \mathrm{p}=.004, \eta^{2}=0.0595\right)$. Breastfeeding parents perceive a significantly higher level of intimacy (Median=5, IQR=5-4) than parents-to-be (Median=4, IQR=5-3; $\mathrm{z}=2.874, \mathrm{p}=.024)$. Negative emotion of breastfeeding is reported as below average at (Median $=2, \mathrm{IQR}=3-1)$ with significant differences between groups $\left(\mathrm{H}_{3}=9.253\right.$, $\left.\mathrm{p}<.001, \eta^{2}=0.1466\right)$. Here, bottle feeding parents have higher negative emotion about breastfeeding (Median $=4, \mathrm{IQR}=5-3)$ than breastfeeding parents $($ Median $=2, \mathrm{IQR}=3-1$; $\mathrm{z}=7.546, \mathrm{p}<.001$ ), partners (Median $=2, \mathrm{IQR}=3-2 ; \mathrm{z}=4.837, \mathrm{p}<.001$ ), and parents-to-be (Median $=3, \mathrm{IQR}=3-3 ; \mathrm{z}=2.641, \mathrm{p}=.049$ ). Parentsto-be also perceive a higher negative emotion than breastfeeding parents with $(\mathrm{z}=5.464, \mathrm{p}<0.001)$. Burden of breastfeeding is reported as average at (Median $=3, \mathrm{IQR}=4-2$ ). A significant difference was found $\left(\mathrm{H}_{3}=13.098, \mathrm{p}=.004, \eta^{2}=0.0294\right)$ between breastfeeding parents (Median=3, IQR=4-2) and parents-to-be (Median=4, IQR=4-3; $\mathrm{z}=3.262, \mathrm{p}=.007)$. Comments show that breastfeeding parents view the feeding process as an opportunity to bond with their child, e.g., "it [breastfeeding] offers intimate child-parent moments, there are plenty of those outside of breastfeeding." (S76. breastfeeding parent). Bottle feeding parents highlighted benefits for partners, but also reported vulnerability and negative emotions as a result of their choice of feeding method, e.g., "[...] breastfeeding is a definite plus specifically for the baby in the first few weeks. But not as much for the mother. I nearly got post natal depression as a consequence of formula feeding stigmatisation" (S136).

Technology deployment in a sensitive setting Participants cautiously agree that technology has potential to support breastfeeding and infant feeding (Median $=4, \mathrm{IQR}=4-3$ ), with no significant differences between groups $\left(\mathrm{H}_{3}=4.538, \mathrm{p}=.209, \eta^{2}=0.0013\right)$. Likewise, attitudes toward technology in this setting are average (Median=3, $\mathrm{IQR}=4-2)$, with no significant differences between groups $\left(\mathrm{H}_{3}=3.161\right.$, $\mathrm{p}=.367, \eta^{2}=0.0002$ ). Qualitative feedback puts these findings in perspective, with respondents being sceptical about the potential of technology to provide practical support, e.g., "practical/physical aspects of breastfeeding that I think limit the usefulness of technology at least in actually learning to breastfeed." (S119, Breastfeeding parent) and "I had logging programs (and even an accurate smart scale!!) but they were not worth the effort" (S201, breastfeeding parent), while having potential as a source of information, e.g., "[I] learned more helpful information from online sources like KellyMom and La Leche League than from my doctor/midwife/gynaecologist combined" (S139, Breastfeeding parent). Participants reported average concerns about technology (Median=3, IQR=4-2). However, there are significant differences between groups $\left(\mathrm{H}_{3}=10.487, \mathrm{p}=.015, \eta^{2}=0.0115\right)$, with breastfeeding parents scoring higher (Median $=3, \mathrm{IQR}=4-2 ; \mathrm{z}=3.203$, $\mathrm{p}=.008$ ) than partners (Median=2, IQR=3-2). Comments reveal concerns related to the credibility of the information, e.g., "Tech is only the vehicle, the real issue is the quality of information" (S134, breastfeeding parent).

\subsection{Summary of Findings}

Results generally support findings from the interview study, suggesting that breastfeeding parents consider the activity important, view it in a positive light, have a good understanding thereof, and in consequence experience high agency throughout the feeding process. Compared to bottle feeding parents, the main difference lies in breastfeeding attitude and personal agency, which is substantially lower. Regardless of feeding mode, external factors such as social norms and environment are not always perceived as openly supportive toward breastfeeding, leaving room for improvement. In terms of becoming a resilient infant feeder, findings support the notion that breastfeeding is a complex and surprisingly challenging process that threatens perceived autonomy particularly in the beginning of the feeding relationship. In contrast to interview findings, survey respondents did not report pressure to breastfeed from family and friends, but were aware of perspectives of healthcare professionals as well as wider expectations of society on whether, how and where to breastfeed. In terms of the emotional toll, there was consensus that breastfeeding is an opportunity to bond with one's child, but parents who did not breastfeed experienced negative emotions including feelings of loss and guilt. Finally, the use of 
technology as an information source in the early stages of breastfeeding was widely accepted and results highlight the potential to help parents seek peer support. However, parents were not convinced that technology could also address the practical challenges of breastfeeding, echoing interviewees' concerns.

\section{DISCUSSION}

Here, we summarise key findings with respect to our initial research questions, and outline points for reflection for technology to support breastfeeding.

RQ1: What is parents' anticipated or lived experience of infant feeding, and what are implications for the role of technology to support breastfeeding? Our results highlight that the lived experience of breastfeeding is messier than what parents anticipate. In particular, parents highlighted the impact of practical and emotional challenges throughout the feeding journey, e.g., having to make ad-hoc adjustments to the feeding process in response to problems, or having to embrace the lack of routine that can come with breastfeeding. Additionally, our results highlight that not all factors that influence the lived experience reside within the breastfeeding parent, but are associated with their environment (e.g., wider society or the built environment). This suggests that technology directly addressing parents needs to embrace and adequately reflect the complexities of breastfeeding to facilitate realistic expectation setting at the prenatal stage, and flexible support throughout the feeding process. However, rather than solely focusing on parents, our findings suggest that there also is potential for technology interventions targeting wider society, e.g., to raise awareness of breastfeeding parents' needs and to further normalise breastfeeding.

RQ2: What are parents' general perspectives on technology to support breastfeeding, how do they align with their preferences and needs across the feeding journey? Participants were cautiously optimistic about the potential of technology to support breastfeeding, suggesting that they would be willing to engage with technology-based support (or already do so). However, the participants were sceptical about the potential of technology in providing practical support. Considering the breastfeeding journey, it is important to recognise changing parental needs, e.g., the wide adoption of tracking technology is viewed as trivial in later postnatal stages once a feeding routine has been established. Furthermore, concerns about the credibility of information highlight the delicate nature of infant feeding, suggesting demand for supporting interventions endorsed by organisations and policymakers.

\subsection{The Relevance of the Lived Experience When Designing Technology to Support Breastfeeding}

Our work highlights the importance for technology that seeks to support breastfeeding to appreciate the complexity of the lived experience of infant feeding. While results of the IBM show that breastfeeding success is associated with the importance and benefits of the activity along with perceived agency, our findings regarding the lived experience further illustrate how parents view the process of breastfeeding, which can be complicated, bring up conflicting emotions, and requires parents to be resilient in the light of highly individual challenges. However, dedicated technology to support breastfeeding developed by the research community predominantly focuses on education by conveying factual knowledge with little reference to the lived experience [65], risking to present the activity in a mono-dimensional way rather than reflecting its multifaceted reality. Interestingly, technology use by parents as reported in our research suggests that unspecific systems (e.g., spreadsheets) are routinely leveraged in a bid to manage the feeding process and re-gain agency (e.g., by reading about other people's challenges or tracking feeding routines), suggesting that it plays a role in helping parents manage messiness and complexity, something which is also echoed by commercial systems (e.g., baby tracking apps [51]) and online resources (e.g., KellyMom [10]) that cater to these needs.

Hence, technology to support breastfeeding needs to focus on how to adequately communicate the lived experience, how to further help parents manage complexity and build resilience, while also recognising their individual experiences throughout the feeding journey. For systems targeting parents in the prenatal stages, this means that choice of technology is not as important as the fact that content needs to be carefully crafted so that it accurately conveys the complexity of lived breastfeeding experiences (without discouraging breastfeeding). We speculate that interactive simulations might be used to portray the lived experience, e.g., by means of mobile applications that offer interactive insights into breastfeeding, or games as suggested by [32]. For systems to be used in the postnatal period, the focus should be on supporting parental resilience. Here, systems should provide tailored and reliable information on potential challenges; designers need to ensure that the content is aligned with official guidelines and endorsed by trustworthy authorities. In terms of deployment platform, our data suggests that easily accessible platforms such as mobile apps or websites are most suited for a period during which parents typically have few hands to spare. However, we would also like to note that there are issues that cannot (and should not) be addressed through technology (e.g., if a parent experiences serious challenges in the breastfeeding process). Here, access to human support is vital, and technology needs to move into the background (e.g., serving as a platform to connect relevant parties).

\subsection{Casting Breastfeeding as Collective Rather Than Individual Effort}

Our results suggest that social and environmental factors have a strong impact on breastfeeding practice, while the labour of carrying though with breastfeeding is usually placed on the individual [38]. Likewise, technology in this space predominantly focuses on mothers [65], and even in instances where negative implications of societal and environmental aspects are recognised, burden is still placed on the breastfeeding parent (e.g., in the application FeedFinder [6] that helps breastfeeding parents identify breastfeeding-friendly spaces). While an effective workaround in the short term, such systems run the risk of distracting from the fact that breastfeeding support should be a collective effort, where family and wider society also carry responsibility [56]. Therefore, technology needs to adopt a broader perspective on breastfeeding support, and not only address the breastfeeding parent, but also involve partners and their families along with wider society in an 
effort to better support breastfeeding parents. In terms of opportunities for technology to support this change, we see potential in systems that can help other parties understand the lived experience of breastfeeding, and systems that highlight the needs of breastfeeding parents. For example, text message or notification systems that have typically been used to target breastfeeding mothers could also be leveraged to encourage partners and family members to actively get involved and help breastfeeding persons. Beyond the involvement of immediate family, there is an opportunity to develop systems that communicate requirements for breastfeeding-friendly spaces to policy makers, architects, and employers, allowing them to reflect on their design choices through the lens of breastfeeding parents. For example, we envision a VR simulation that immerses the stakeholders in a breastfeeding (or milk expression) experience taking place in the model of an office building. Likewise, there is potential to leverage technology to normalise breastfeeding in wider society, increasing the awareness of the feeding process and parents' right to public breastfeeding. Thereby, we hope that technology can support a shift from individual to collective responsibility for successful breastfeeding, rather than reinforcing existing structures that place the burden on the breastfeeding parent.

\section{LIMITATIONS AND FUTURE WORK}

There are a number of limitations and opportunities for future research associated with the research presented in this paper. Most importantly, we would like to reflect on the participant samples that we drew for the online survey in particular. Many respondents were successful longer-term breastfeeding parents rather than parents who at some point decided to bottle feed. The sample of bottle feeding parents and responses from partners were more limited. In the interview study, we did not specifically investigate the role of lactation consultants as this service is frequently provided by midwives in the region where our work was carried out. However, in other geographical regions, exploring their role in particular is important due to the support they provide to new parents. While these limitations do not limit the implications of our findings for the group of breastfeeding parents, a further exploration of the other groups along with an examination of breastfeeding in additional countries might provide valuable further insights into barriers toward breastfeeding and how to better support parents. In addition, our research built on technology participants already used rather than the introduction and discussion of new artefacts, and our choice on the theoretical model does not reflect the impact of individuals' feeding experience on breastfeeding determinants. Likewise, our work does not especially investigate the impact of the child's age and the maturity of feeding relationship on the feeding experience. In the future, such an investigation could supplement our work, as our initial findings already suggest that the perception of breastfeeding and support needs change across the feeding journey.

\section{CONCLUSION}

Technology has the potential to support breastfeeding. However, little research has explored the points of reflection for technology design to support breastfeeding through the perspectives of different stakeholders in infant feeding space. Our work leveraged the IBM to inquire the lived and anticipated experiences of breastfeeding and the perspectives toward technology to support breastfeeding from stakeholders in infant feeding space. The results show a complex lived breastfeeding experience with predominant challenges in practical, emotional, environmental and societal settings, in which technology could play a role in supporting parents. Our work lays out challenges and implications for technology design to support breastfeeding, enabling researchers and technology designers to contribute to this space through the development of technology to support parents.

\section{ACKNOWLEDGMENTS}

We would like to thank all interview and survey participants for sharing their feeding experiences with us. We very much appreciate your openness and time. We would also like to thank KU Leuven Campus Group T for funding this work through a PhD scholarship for the main author.

\section{REFERENCES}

[1] Jennifer Abbass-Dick, Michele Brolly, Joanne Huizinga, Amber Newport, Fangli Xie, Stephanie George, and Elisabeth Sterken. 2018. Designing an eHealth Breastfeeding Resource With Indigenous Families Using a Participatory Design. fournal of Transcultural Nursing: Official fournal of the Transcultural Nursing Society 29, 5 (2018), 480-488. https://doi.org/10.1177/1043659617731818

[2] Indu B. Ahluwalia, Brian Morrow, and Jason Hsia. 2005. Why do women stop breastfeeding? Findings from the Pregnancy Risk Assessment and Monitoring System. Pediatrics 116, 6 (Dec 2005), 1408-1412. https://doi.org/10.1542/peds. 2005-0013

[3] Icek Ajzen. 1985. From Intentions to Actions: A Theory of Planned Behavior. Springer Berlin Heidelberg, Berlin, Heidelberg, 11-39. https://doi.org/10.1007/ 978-3-642-69746-3_2

[4] Nazanin Andalibi and Andrea Forte. 2018. Announcing Pregnancy Loss on Facebook: A Decision-Making Framework for Stigmatized Disclosures on Identified Social Network Sites. In Proceedings of the 2018 CHI Conference on Human Factors in Computing Systems (Montreal QC, Canada) (CHI '18). Association for Computing Machinery, New York, NY, USA, 1-14. https://doi.org/10.1145/3173574. 3173732

[5] Samir Arora, Cheryl McJunkin, Julie Wehrer, and Phyllis Kuhn. 2000. Major factors influencing breastfeeding rates: Mother's perception of father's attitude and milk supply. Pediatrics 106, 5 (Nov 2000), E67. https://doi.org/10.1542/peds. 106.5.e67

[6] Madeline Balaam, Rob Comber, Ed Jenkins, Selina Sutton, and Andrew Garbett. 2015. FeedFinder: A Location-Mapping Mobile Application for Breastfeeding Women. In Proceedings of the 33rd Annual ACM Conference on Human Factors in Computing Systems (Seoul, Republic of Korea) (CHI '15). Association for Computing Machinery, New York, NY, USA, 1709-1718. https: //doi.org/10.1145/2702123.2702328

[7] Albert Bandura. 1986. Social foundations of thought and action: A social cognitive theory. Prentice-Hall, Inc, Englewood Cliffs, NJ, US.

[8] Ingrid Blixt, Lena B. Mårtensson, and Anette C. Ekström. 2014. Process-oriented training in breastfeeding for health professionals decreases women's experiences of breastfeeding challenges. International Breastfeeding fournal 9 (2014), 15. https://doi.org/10.1186/1746-4358-9-15

[9] Rosemary Blyth, Debra K. Creedy, Cindy-Lee Dennis, Wendy Moyle, Jan Pratt, and Susan M. De Vries. 2002. Effect of maternal confidence on breastfeeding duration: an application of breastfeeding self-efficacy theory. Birth (Berkeley, Calif.) 29, 4 (Dec 2002), 278-284.

[10] Kelly Bonyata. 2020. Kellymom-Parenting Breastfeeding. https://kellymom.com/.

[11] Richard E. Boyatzis. 1998. Transforming qualitative information: Thematic analysis and code development. Sage Publications, Inc, Thousand Oaks, CA, US. xvi, 184 pages.

[12] Kate Boyer. 2011. "The way to break the taboo is to do the taboo thing" breastfeeding in public and citizen-activism in the UK. Health \& Place 17, 2 (Mar 2011), 430-437. https://doi.org/10.1016/j.healthplace.2010.06.013

[13] Robert E. Braun, Tavis Glassman, Jiunn-Jye Sheu, Joseph Dake, Tim Jordan, and Faith Yingling. 2014. Using the Integrated Behavioral Model to Predict High-Risk Drinking among College Students. Fournal of Alcohol and Drug Education 58, 2 (Aug 2014), 46-63.

[14] Virginia Braun and Victoria Clarke. 2006. Using thematic analysis in psychology. Qualitative Research in Psychology 3, 2 (Jan 2006), 77-101. https://doi.org/10. 1191/1478088706qp063oa 
[15] Virginia Braun and Victoria Clarke. 2012. Thematic analysis. American Psychological Association, Washington, DC, US, 57-71. https://doi.org/10.1037/13620-004

[16] P. Chaovalit and S. Pongnumkul. 2017. Moommae: A location-aware mobile information system for breastfeeding mothers in Thailand (invited paper). In 2017 10th International Conference on Ubi-media Computing and Workshops (Ubi-Media) IEEE, Pattaya, Thailand, 1-5. https://doi.org/10.1109/UMEDIA.2017.8074104

[17] Erika Chin, Adrienne Porter Felt, Vyas Sekar, and David Wagner. 2012. Measuring User Confidence in Smartphone Security and Privacy. In Proceedings of the Eighth Symposium on Usable Privacy and Security (Washington, D.C.) (SOUPS '12) Association for Computing Machinery, New York, NY, USA, Article 1, 16 pages. https://doi.org/10.1145/2335356.2335358

[18] Barry H. Cohen. 2008. Explaining Psychological Statistics. John Wiley \& Sons, Hoboken, New Jersey.

[19] D. R. dela Cruz and D. M. M. Mendoza. 2017. Milktrack: Design and development of mobile application and logistics system in empowering breastfeeding practice in the Philippines. In TENCON 2017 - 2017 IEEE Region 10 Conference. IEEE, Penang, Malaysia, 2242-2246. https://doi.org/10.1109/TENCON.2017.8228234

[20] Jill Demirci, Virginia Kotzias, Debra L. Bogen, Kristin N. Ray, and Lori UscherPines. 2019. Telelactation via Mobile App: Perspectives of Rural Mothers, Their Care Providers, and Lactation Consultants. Telemedicine fournal and E-Health: The Official fournal of the American Telemedicine Association 25, 9 (Sep 2019), 853-858. https://doi.org/10.1089/tmj.2018.0113

[21] Jill R. Demirci, Brian Suffoletto, Jack Doman, Melissa Glasser, Judy C. Chang Susan M. Sereika, and Debra L. Bogen. 2020. The Development and Evaluation of a Text Message Program to Prevent Perceived Insufficient Milk Among First-Time Mothers: Retrospective Analysis of a Randomized Controlled Trial. $7 \mathrm{MIR}$ mHealth and uHealth 8, 4 (2020), e17328. https://doi.org/10.2196/17328

[22] Catherine D'Ignazio, Alexis Hope, Becky Michelson, Robyn Churchill, and Ethan Zuckerman. 2016. A Feminist HCI Approach to Designing Postpartum Technologies: "When I First Saw a Breast Pump I Was Wondering If It Was a Joke". In Proceedings of the 2016 CHI Conference on Human Factors in Computing Systems (San Jose, California, USA) (CHI '16). Association for Computing Machinery, New York, NY, USA, 2612-2622. https://doi.org/10.1145/2858036.2858460

[23] Susan M. Donath, Lisa Helen Amir, and ALSPAC Study Team. 2003. Relationship between prenatal infant feeding intention and initiation and duration of breastfeeding: a cohort study. Acta Paediatrica (Oslo, Norway: 1992) 92, 3 (2003), 352-356.

[24] Olive Jean Dunn. 1961. Multiple Comparisons among Means. F. Amer. Statist Assoc. 56, 293 (Mar 1961), 52-64. https://doi.org/10.1080/01621459.1961.10482090

[25] Roger A. Edwards, Timothy Bickmore, Lucia Jenkins, Mary Foley, and Justin Manjourides. 2013. Use of an interactive computer agent to support breastfeeding. Maternal and Child Health fournal 17, 10 (Dec 2013), 1961-1968. https://doi.org/ 10.1007/s10995-013-1222-0

[26] Morten W. Fagerland. 2012. t-tests, non-parametric tests, and large studies-a paradox of statistical practice? BMC Medical Research Methodology 12, 1 (Jun 2012), 78. https://doi.org/10.1186/1471-2288-12-78

[27] Martin Fishbein. 1979. A theory of reasoned action: Some applications and implications. Nebraska Symposium on Motivation 27 (1979), 65-116.

[28] William A. Fisher, Jeffrey D. Fisher, and Jennifer Harman. 2003. The InformationMotivation-Behavioral Skills Model: A General Social Psychological Approach to Understanding and Promoting Health Behavior. John Wiley \& Sons, Ltd, Malden, Massachusetts, Chapter 4, 82-106. https://doi.org/10.1002/9780470753552.ch4 arXiv:https://onlinelibrary.wiley.com/doi/pdf/10.1002/9780470753552.ch4

[29] Carol A. Friesen, Laura J. Hormuth, Devan Petersen, and Tina Babbitt. 2015 Using Videoconferencing Technology to Provide Breastfeeding Support to LowIncome Women: Connecting Hospital-Based Lactation Consultants with Clients Receiving Care at a Community Health Center. Journal of Human Lactation Official fournal of International Lactation Consultant Association 31, 4 (Nov 2015), 595-599. https://doi.org/10.1177/0890334415601088

[30] Lisa Gatti. 2008. Maternal perceptions of insufficient milk supply in breastfeeding. Fournal of Nursing Scholarship: An Official Publication of Sigma Theta Tau International Honor Society of Nursing 40, 4 (2008), 355-363. https://doi.org/10. 1111/j.1547-5069.2008.00234.x

[31] Nicola Geoghegan-Morphet, Doris Yuen, Esther Rai, Michelle Angelini, Melissa Christmas, and Orlando da Silva. 2014. Development and implementation of a novel online breastfeeding support resource: the Maternal Virtual Infant Nutrition Support Clinic. Breastfeeding Medicine: The Official fournal of the Academy of Breastfeeding Medicine 9, 10 (Dec 2014), 520-523. https://doi.org/10.1089/bfm. 2014.0051

[32] Kathrin Gerling, Kieran Hicks, Laura Buttrick, Chris Headleand, Ben Williams, Jason Hall, Kymeng Tang, Luc Geurts, and Wei Chen. 2018. Potential and Limitations of Playful Technology to Support Infant Feeding. In Proceedings of the 2018 Annual Symposium on Computer-Human Interaction in Play Companion Extended Abstracts (CHI PLAY '18 Extended Abstracts). ACM, New York, NY, USA, 431-437. https://doi.org/10.1145/3270316.3271519

[33] Lorna Gibson and Vicki L. Hanson. 2013. Digital Motherhood: How Does Technology Help New Mothers? In Proceedings of the SIGCHI Conference on Human
Factors in Computing Systems (Paris, France) (CHI '13). Association for Computing Machinery, New York, NY, USA, 313-322. https://doi.org/10.1145/2470654. 2470700

[34] Jane S. Grassley, Kelley C. Connor, and Laura Bond. 2017. Game-based online antenatal breastfeeding education: A pilot. Applied nursing research: ANR 33 (2017), 93-95. https://doi.org/10.1016/j.apnr.2016.10.011

[35] Xinning Gui, Yu Chen, Yubo Kou, Katie Pine, and Yunan Chen. 2017. Investigating Support Seeking from Peers for Pregnancy in Online Health Communities. Proc. ACM Hum.-Comput. Interact. 1, CSCW, Article 50 (Dec. 2017), 19 pages. https: //doi.org/10.1145/3134685

[36] Ashish Joshi, Douglas M. Puricelli Perin, Chioma Amadi, and Kate Trout. 2015. Evaluating the usability of an interactive, bi-lingual, touchscreen-enabled breastfeeding educational programme: application of Nielson's heuristics. Fournal of Innovation in Health Informatics 22, 2 (Mar 2015), 265-274. https://doi.org/10. 14236/jhi.v22i2.71

[37] Danuta Kasprzyk and Daniel E. Montaño. 2007. Application of an Integrated Behavioral Model to Understand HIV Prevention Behavior of High-Risk Men in Rural Zimbabwe. Taylor \& Francis, New York, 149-169. https://doi.org/10.4324/ 9780203937082-17

[38] Christa M. Kelleher. 2006. The physical challenges of early breastfeeding. Social Science \& Medicine 63, 10 (Nov 2006), 2727-2738. https://doi.org/10.1016/j. socscimed.2006.06.027

[39] Jacqueline C. Kent, Elizabeth Ashton, Catherine M. Hardwick, Marnie K. Rowan, Elisa S. Chia, Kyle A. Fairclough, Lalitha L. Menon, Courtney Scott, Georgia Mather-McCaw, and Katherine Navarro. 2015. Nipple Pain in Breastfeeding Mothers: Incidence, Causes and Treatments. International fournal of Environmental Research and Public Health 12, 10 (Oct 2015), 12247-12263. https: //doi.org/10.3390/ijerph121012247

[40] Julia H. Kim, Barbara H. Fiese, and Sharon M. Donovan. 2017. Breastfeeding is Natural but Not the Cultural Norm: A Mixed-Methods Study of First-Time Breastfeeding, African American Mothers Participating in WIC. Fournal of Nutrition Education and Behavior 49, 7, Supplement 2 (Jul 2017), S151-S161.e1. https://doi.org/10.1016/j.jneb.2017.04.003

[41] William H. Kruskal and W. Allen Wallis. 1952. Use of Ranks in One-Criterion Variance Analysis. F. Amer. Statist. Assoc. 47, 260 (1952), 583-621. https://doi. org $/ 10.2307 / 2280779$

[42] José Labarère, Nathalie Gelbert-Baudino, Laurent Laborde, Domitille Arragain, Camille Schelstraete, and Patrice François. 2011. CD-ROM-based program for breastfeeding mothers. Maternal \& Child Nutrition 7, 3 (Jul 2011), 263-272. https://doi.org/10.1111/j.1740-8709.2009.00235.x

[43] Gabriel M. Leung, Tai Hing Lam, and Lai Ming Ho. 2002. Breast-feeding and its relation to smoking and mode of delivery. Obstetrics and Gynecology 99, $5 \mathrm{Pt} 1$ (May 2002), 785-794. https://doi.org/10.1016/s0029-7844(02)01940-3

[44] M. Kay Libbus and Linda S. Kolostov. 1994. Perceptions of Breastfeeding and Infant Feeding Choice in a Group of Low-income Mid-Missouri Women. Journal of Human Lactation 10, 1 (Mar 1994), 17-23. https://doi.org/10.1177/ 089033449401000123

[45] Liam Mason, Kathrin Gerling, Patrick Dickinson, and Antonella De Angeli. 2019. Design Goals for Playful Technology to Support Physical Activity Among Wheelchair Users. In Proceedings of the 2019 CHI Conference on Human Factors in Computing Systems (Glasgow, Scotland Uk) (CHI '19). Association for Computing Machinery, New York, NY, USA, 1-12. https://doi.org/10.1145/3290605.3300262

[46] Deborah McCarter-Spaulding and Margaret H. Kearney. 2001. Parenting selfefficacy and perception of insufficient breast milk. fournal of obstetric, gynecologic, and neonatal nursing: 7OGNN 30, 5 (Oct 2001), 515-522. https://doi.org/10.1111/ j.1552-6909.2001.tb01571.x

[47] Shahla Meedya, Kathleen Fahy, and Ashley Kable. 2010. Factors that positively influence breastfeeding duration to 6 months: a literature review. Women and Birth: Fournal of the Australian College of Midwives 23, 4 (Dec 2010), 135-145. https://doi.org/10.1016/j.wombi.2010.02.002

[48] Amal K. Mitra, Amal J. Khoury, Agnes W. Hinton, and Cathy Carothers. 2004. Predictors of breastfeeding intention among low-income women. Maternal and Child Health fournal 8, 2 (Jun 2004), 65-70.

[49] Daniel Montaño and Kasprzyk Danuta. 1992. Health Behavior and Health Education: Theory, Research, and Practice. Annals of Internal Medicine 116, 4 (Feb 1992), 350. https://doi.org/10.7326/0003-4819-116-4-350

[50] Candace Mulready-Ward and Martine Hackett. 2014. Perception and Attitudes: Breastfeeding in Public in New York City. Journal of Human Lactation 30, 2 (May 2014), 195-200. https://doi.org/10.1177/0890334414524988

[51] Nighp. 2015. Nighp-Baby Tracker. http://www.nighp.com/babytracker/

[52] Gary Ong, Mabel Yap, Foo Ling Li, and Tai Bee Choo. 2005. Impact of working status on breastfeeding in Singapore: evidence from the National Breastfeeding Survey 2001. European fournal of Public Health 15, 4 (Aug 2005), 424-430. https: //doi.org/10.1093/eurpub/cki030

[53] World Health Organization. 2017. WHO | Tracking progress for breastfeeding policies and programmes: Global breastfeeding scorecard 2017. http://www. who.int/nutrition/publications/infantfeeding/global-bf-scorecard-2017/en/ 
[54] World Health Organization. 2019. WHO | Breastfeeding. https://www.who.int/ health-topics/breastfeeding

[55] Keiko Otsuka, Cindy-Lee Dennis, Hisae Tatsuoka, and Masamine Jimba. 2008 The relationship between breastfeeding self-efficacy and perceived insufficient milk among Japanese mothers. Journal of obstetric, gynecologic, and neonatal nursing: FOGNN 37, 5 (Oct 2008), 546-555. https://doi.org/10.1111/j.1552-6909. 2008.00277.x

[56] Rachel Pain, Cathy Bailey, and Graham Mowl. 2001. Infant feeding in North East England: contested spaces of reproduction. Area 33, 3 (Sep 2001), 261-272. https://doi.org/10.1111/1475-4762.00030

[57] James O. Prochaska and Wayne F. Velicer. 1997. The Transtheoretical Model of Health Behavior Change. American fournal of Health Promotion 12, 1 (Sep 1997), 38-48. https://doi.org/10.4278/0890-1171-12.1.38

[58] Enrica Riva, Giuseppe Banderali, Carlo Agostoni, Marco Silano, Giovanni Radaelli, and Marcello Giovannini. 1999. Factors associated with initiation and duration of breastfeeding in Italy. Acta Paediatrica 88, 4 (1999), 411-415. https://doi.org/ 10.1111/j.1651-2227.1999.tb01132.x

[59] Wilaiporn Rojjanasrirat, Eve-Lynn Nelson, and Karen A. Wambach. 2012. A pilot study of home-based videoconferencing for breastfeeding support. Fournal of Human Lactation: Official fournal of International Lactation Consultant Association 28, 4 (Nov 2012), 464-467. https://doi.org/10.1177/0890334412449071

[60] Nigel C Rollins, Nita Bhandari, Nemat Hajeebhoy, Susan Horton, Chessa K Lutter Jose C Martines, Ellen G Piwoz, Linda M Richter, and Cesar G Victora. 2016. Why invest, and what it will take to improve breastfeeding practices? The Lancet 387 , 10017 (Jan 2016), 491-504. https://doi.org/10.1016/S0140-6736(15)01044-2

[61] Irwin M. Rosenstock, Victor J. Strecher, and Marshall H. Becker. 1988. Social learning theory and the Health Belief Model. Health Education Quarterly 15, 2 (1988), 175-183.

[62] Richard M. Ryan and Edward L. Deci. 2000. Self-determination theory and the facilitation of intrinsic motivation, social development, and well-being. American Psychologist 55, 1 (2000), 68-78. https://doi.org/10.1037/0003-066X.55.1.68

[63] Stephanie Sayres and Lisa Visentin. 2018. Breastfeeding: uncovering barriers and offering solutions. Current Opinion in Pediatrics 30, 4 (2018), 591-596. https //doi.org/10.1097/MOP.0000000000000647
[64] Jane A. Scott, Maeran C. Landers, Robert M. Hughes, and Colin W. Binns. 2001. Factors associated with breastfeeding at discharge and duration of breastfeeding. fournal of Paediatrics and Child Health 37, 3 (Jun 2001), 254-261. https://doi.org/ 10.1046/j.1440-1754.2001.00646.x

[65] Kymeng Tang, Kathrin Gerling, Wei Chen, and Luc Geurts. 2019. Information and Communication Systems to Tackle Barriers to Breastfeeding: Systematic Search and Review. Journal of Medical Internet Research 21, 9 (2019), e13947. https://doi.org/10.2196/13947

[66] Chih-Jau Wang, Pimwadee Chaovalit, and Suporn Pongnumkul. 2018. A Breastfeed-Promoting Mobile App Intervention: Usability and Usefulness Study. FMIR mHealth and uHealth 6, 1 (2018), e27. https://doi.org/10.2196/mhealth.8337

[67] Chelsea-Joy Wardle, Mitchell Green, Christine Wanjiru Mburu, and Melissa Densmore. 2018. Exploring Co-Design with Breastfeeding Mothers. In Proceedings of the 2018 CHI Conference on Human Factors in Computing Systems (Montreal QC, Canada) (CHI '18). Association for Computing Machinery, New York, NY, USA, 1-12. https://doi.org/10.1145/3173574.3174056

[68] Becky K. White, Roslyn C. Giglia, Jane A. Scott, and Sharyn K. Burns. 2018. How New and Expecting Fathers Engage With an App-Based Online Forum: Qualitative Analysis. 7MIR mHealth and uHealth 6, 6 (Jun 2018), e144. https: //doi.org/10.2196/mhealth.9999

[69] Iain Williamson, Dawn Leeming, Steven Lyttle, and Sally Johnson. 2012. 'It should be the most natural thing in the world': exploring first-time mothers' breastfeeding difficulties in the UK using audio-diaries and interviews. Maternal \& Child Nutrition 8, 4 (2012), 434-447. https://doi.org/10.1111/j.1740-8709.2011. 00328.x

[70] Deepika Yadav, Prerna Malik, Kirti Dabas, and Pushpendra Singh. 2019. Feedpal: Understanding Opportunities for Chatbots in Breastfeeding Education of Women in India. Proceedings of the ACM on Human-Computer Interaction 3, CSCW (Nov 2019), 170:1-170:30. https://doi.org/10.1145/3359272

[71] Moleen Zunza, Mark F Cotton, Lawrence Mbuagbaw, Richard Lester, and Lehana Thabane. 2017. Interactive weekly mobile phone text messaging plus motivational interviewing in promotion of breastfeeding among women living with HIV in South Africa: study protocol for a randomized controlled trial. Trials $18,1 \mathrm{Ju}$ 2017), 331. https://doi.org/10.1186/s13063-017-2079-0 